\title{
IDENTAL
}

\section{Management of chronic periodontal diseases within the NHS}

\author{
I.L.C. Chapple ${ }^{1}$
}

\begin{abstract}
Studies estimate that up to $60 \%$ of tooth loss results from periodontal disease. Oral health awareness amongst the public has increased dramatically over the last decade and patients now place a great deal of importance upon tooth retention. Given the potential for premature mortality due to cardiovascular disease, the '20per-day' smoker may contemplate giving up in 'the future.' However, when faced with the loss of an anterior tooth, they will contemplate immediate cessation and many succeed because the immediate consequences of not doing so are very tangible and personal.
\end{abstract}

In 2001 the $B D J$ published the results of the 1998 Adult Dental Health Survey, where Morris and co-workers reported severe destructive periodontitis affected $8 \%$ of the population, with 54\% of adults having some pocketing. More worryingly 34\% of 16-24 year olds had some pocketing and $14 \%$ had attachment loss! The Dental Practice Board statistics for 2001/2 indicated that £174 million was spent on 15.5 million treatment episodes of NHS periodontal therapy alone: $89 \%$ were item 10a, 8.4\% item $10 \mathrm{~b}$ and 2.6\% (400,000 treatment episodes) item 10c. Assuming a UK population of 60 million, a 50\% dental attendance rate, and a periodontal disease prevalence of $10 \%$ of the population, then 3 million dental attendees will suffer from significant periodontal disease. However, only $2.6 \%$ of treatment episodes fell under item 10c, the category designated for managing patients with significant periodontal disease, which amounts to only 13\% of those with the disease!

In the same year (2001) Morgan reported the results of a practice-based QA audit of 464 clinical records and demonstrated that $20 \%$ had some form of periodontal screening and only $1.5 \%$ had any detailed periodontal assessment. It would appear that

${ }^{1}$ Professor, University of Birmingham, Dental School, St Chad's Queensway, Birmingham B4 6NN Correspondence to: Professor I.L.C. Chapple Email: i.l.c.chapple@bham.ac.uk

\section{doi:10.1038/sj.bdj.4811180}

๑ British Dental Journal 2004; 196: 505
This article highlights the paper being presented at 09.30 on Friday, 7 May 2004 in the Bourne Lounge at The British Dental Conference \& Exhibition 2004 being held at the Bournemouth International Centre

periodontal diseases are being under-managed within primary care dentistry, an observation that fits with the increased referral rates to specialist services. With that in mind, we set out to examine what barriers existed to periodontal therapy in the primary care sector. We sent a case study of mild chronic periodontitis (CRP) that would be amenable to management in general dental practice to 865 GDPs within the Midlands (64\% responded) and to 59 specialist practitioners (79\% responded). The key factors to emerge were:

- 47\% of GDPs felt unable to allocate the time they believed was necessary to manage the case in practice, due to hopelessly inadequate NHS fees (c.a. £100 per case) for such patients (Time \& Money).

- $80 \%$ of GDPs also under-estimated the time required to manage the case in an 'ideal world', relative to specialist practitioners (Education).

How do we manage this issue in a health service within which we have an aging population, with a greater desire for tooth retention? The principles must be based upon a combination of:

- Increasing resources to manage periodontal diseases within the NHS
- Focussing the limited resources available on those who most need them.

- More cost effective and efficient delegation to other team members.

- Educating the team about the disease process and its management.

The education process must begin with the identification of patients at risk from periodontal diseases and the implementation of rigorous screening protocols for early diagnosis. It is far cheaper to prevent disease progression than to try to manage an established disease.

When should we start screening for chronic periodontal diseases? Convincing data from Clerehugh and colleagues in the 1990s demonstrated that CRP-associated attachment loss starts between 14-19 years of age and is significantly associated with the presence of sub-gingival calculus at 14 years of age. Therefore, we should use codes 0,1 and 2 of the BPE screen for 7-11 year olds and from 12 years upwards we should use the full BPE screen on first molar teeth and lower incisors (those teeth most at risk) until adulthood, when the full BPE should be used.

In summary, chronic periodontal diseases represent an under-stated and underresourced problem in NHS dentistry and more education, time and money are needed to address this growing health care burden.

The British Dental Conference \& Exhibition 2004 is being held at the Bournemouth International Centre between Thursday 6 th and Saturday 8th May 2004

Contact: DMS (Delegate Management Services) for further information:

Tel: 08701666625 or +44 (0) 1252771425

Fax: 08705228890 or +44 (0) 1252771790

For the latest update on the agenda and to download the programme visit: www.bda-events.org 